\title{
Dermatological manifestations of patients on hemodialysis: a longitudinal descriptive study of 208 cases in Dakar
}

\author{
Maodo Ndiaye', Saer Diadie', Ameth Lemrabot Tall², Marie Antoinette Dione', \\ Moussa Diallo', Assane Diop³, Boubacar Ahy Diatta', Ndeye Bougoul Seck', \\ Salimatou Diallo', Mame Téné Ndiaye ${ }^{3}$, Fary Ka², Suzanne Oumou Niang ${ }^{1}$, Fatimata Ly³, \\ Assane Kane', Mame Thierno Dieng ${ }^{1}$
}

${ }^{1}$ Department of Dermatology Aristide Le Dantec Hospital, Cheikh Anta DIOP University of Dakar, Senegal, ${ }^{2}$ Department of Nephrology Aristide le Dantec Hospital, Cheikh Anta DIOP University of Dakar, Sénégal, ${ }^{3}$ Department of Dermatology IHS Dakar, Cheikh Anta DIOP University of Dakar, Senegal

Corresponding author: Dr. Saer Diadie, E-mail: diadie_saer@yahoo.fr

\begin{abstract}
Background: The aim of our study was to assess the prevalence, their clinical and evolutionary aspects and determine their risk of occurrence among Patients on Hemodialysis. Methodology: A descriptive longitudinal study of 15 months, was carried out in five dialysis centers in Dakar. All patient with dermatological manifestations and a dialysis period of 3 weeks or morer were included. Results: 346 dialysis patients were identified among them 208 had dermatological manifestations that means $60.11 \% .89$ women and 119 men with a sex ratio of 1.8 . The mean age was 47.23 years. A personal history of atopy was observed in 41 cases (19.7\%). The most common dermatological manifestation was pruritus (60.5\%), followed by nail disorders (21.6\%), cutaneous xerosis (21.15\%) and pigmentary disorders (15.8\%). There was a statistically significant correlation between pruritus and the reduced number of hemodialysis sessions, the recent nature of dialysis sessions and the existence of personal atopy. A statistically significant correlation was also found between the pigmentary disorders and length of dialysis. Etiologically, the nephroangiosclerosis was the most common cause of nephropathy (31.3\%), followed by diabetic nephropathy (5.8\%). Treatment was symptomatic with antihistamines, emollients and neuroleptics. The response to treatment was satisfactory in half of the patients. Conclusion: Dermatologic manifestations are frequent and polymorphic in dialysis patients. Pruritus by far is the most common.
\end{abstract}

Key words: Skin; Dialys; Senegal

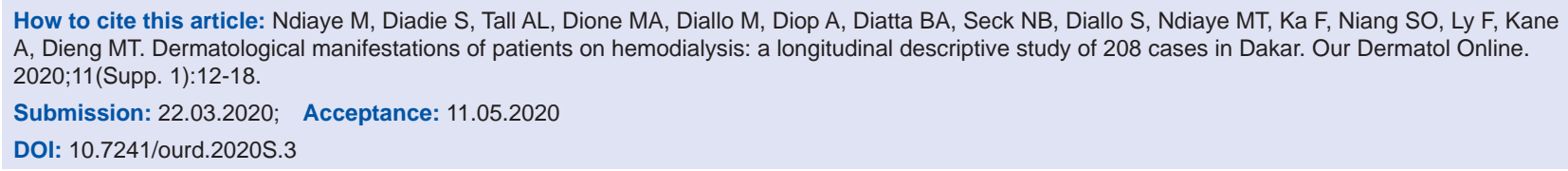




\title{
Les manifestations dermatologiques chez les patients dialysés: étude longitudinale et descriptive de 208 cas à Dakar
}

\author{
Maodo Ndiaye 1 , Saer Diadie ${ }^{1}$, Ameth Lemrabot Tall², Marie Antoinette Dione', \\ Moussa Diallo', Assane Diop ${ }^{3}$, Boubacar Ahy Diatta', Ndeye Bougoul Seck', \\ Salimatou Diallo', Mame Téné Ndiaye³, Fary Ka², Suzanne Oumou Niang ${ }^{1}$, Fatimata Ly³, \\ Assane Kane', Mame Thierno Dieng ${ }^{1}$
}

${ }^{1}$ Department of Dermatology Aristide Le Dantec Hospital, Cheikh Anta DIOP University of Dakar, Senegal, ${ }^{2}$ Department of Nephrology Aristide le Dantec Hospital, Cheikh Anta DIOP University of Dakar, Sénégal, ${ }^{3}$ Department of Dermatology IHS Dakar, Cheikh Anta DIOP University of Dakar, Senegal

Corresponding author: Dr. Saer Diadie, E-mail: diadie_saer@yahoo.fr

\begin{abstract}
RÉSUMÉ
Introduction: les objectifs de notre travail étaient d'évaluer la fréquence des dermatoses chez les dialysés, de déterminer leurs aspects cliniques, leurs facteurs de risque de survenue et leurs aspects évolutifs. Méthodologie: une étude longitudinale descriptive de 15 mois, était réalisée dans cinq centres de dialyse de la ville de Dakar. Ont été inclus, les patients dialysés chroniques depuis 3 semaines et ayant des manifestations dermatologiques. Résultats: nous avons recensé 346 patients dialysés dont 208 présentaient des manifestations dermatologiques soit 60,11\%. Ils se répartissaient en 119 hommes et 89 femmes soit un sex-ratio de 1,8. Lầge moyen était de 47,23 ans. Une atopie personnelle était notée dans 41 cas (19,7\%). Les manifestations dermatologiques étaient dominées par le prurit (60,5\%), suivies par les anomalies unguéales (21,6\%), la xérose cutanée (21,15\%) et les troubles de la pigmentation $(15,8 \%)$. Il y avait une corrélation statistiquement significative entre le prurit et le nombre réduit de séances d'hémodialyse, le caractère récent des séances de dialyse et l'existence d'atopie personnelle. Une corrélation statistiquement significative a été également notée entre les troubles de la pigmentation et l'ancienneté de la dialyse. Sur le plan étiologique, la néphroangiosclérose était la néphropathie causale la plus fréquente $(31,3 \%)$, suivie de la néphropathie diabétique (5,8\%). La prise en charge était symptomatique par les antihistaminiques, les émollients et les neuroleptiques. Lévolution sous traitement était favorable chez la moitié des malades. Conclusion: les manifestations dermatologiques sont fréquentes et polymorphes chez les patients dialysés. Elles sont dominées de loin par le prurit.
\end{abstract}

Mots clés: Peau; Dialyse; Sénégal

\section{INTRODUCTION}

Durant les cinquante dernières années, des progrès ont été notés dans la prise en charge des néphropathies chroniques par la dialyse. Cependant l'apparition de complications multiviscérales (cardiovasculaires, infectieuses, ostéo-articulaires, hématologiques, neurologiques, endocriniennes etc....) altère la qualité de survie obtenue grâce à la dialyse. Ainsi la peau n'est pas épargnée et son atteinte revêt des degrés de gravité variable, allant du simple inconfort à la complication sévère.
L'association de lésions cutanées à l'insuffisance rénale chronique (IRC) a été notée depuis 1827 par Bright [1]. Depuis l'instauration de l'hémodialyse dans les années 1960, les manifestations cutanées sont de plus en plus étudiées. En fait, les dermatoses chez les dialysés chroniques sont fréquentes et variées. Certaines sont peu spécifiques, comme le prurit, les troubles pigmentaires et phanèriens. D'autres sont plus spécifiques mais plus rares, comme les troubles de la kératinisation et la pseudo-porphyrie cutanée tardive.

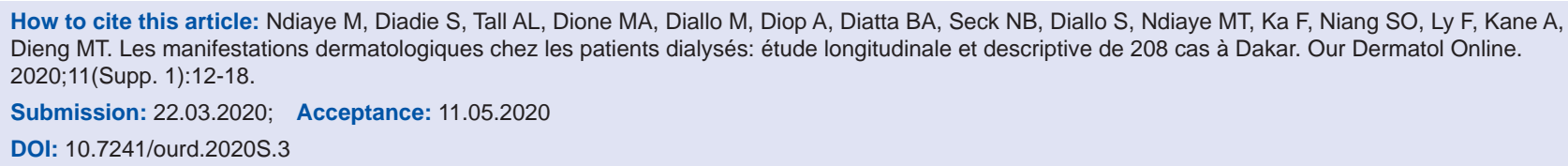


Leur incidence varie de 50 à $100 \%$ des cas selon les séries [2,3]. En Afrique, les études réalisées au Nigéria et au Maroc rapportent respectivement une fréquence de $89,1 \%$ [4] et de $100 \%$ [5].

Au Sénégal, jusqu'à ce jour, aucune étude portant sur les manifestations cutanées chez les dialysés n'a été réalisée. Les objectifs de notre travail étaient d'évaluer la fréquence des dermatoses chez les dialysés, et de déterminer leurs aspects cliniques, leurs facteurs de risque de survenue et leurs aspects évolutifs.

\section{MÉTHODOLOGIE}

Il s'agit d'une étude longitudinale descriptive de 15 mois, réalisée d'Avril 2018 à Juin 2019 dans cinq centres de dialyse de la ville de Dakar (Unité d'hémodialyse et Unité de dialyse péritonéale de l'hôpital Aristide Le Dantec, Unité d'hémodialyse de l'Institut Clinique de Perfectionnement, Unité d'hémodialyse du Centre de diagnostic et d'exploration et Unité d'hémodialyse de la Clinique $\mathrm{ABC}$ ). Ont été inclus dans cette étude, les patients dialysés chroniques depuis 3 semaines dans les différents centres cités précédemment et ayant des manifestations dermatologiques. L'hémodialyse chronique était prescrite par le néphrologue à raison de 3 séances par semaine de 4 heures chacune. Cependant, certains malades en faisaient 1 ou 2 selon les moyens financiers. La membrane de dialyse était en polysulfone. Lanticoagulant utilisé était l'héparine standard. La température du dialysat était de $37^{\circ} \mathrm{C}$ et la concentration du calcium de $1,75 \mathrm{mmol} / \mathrm{l}$. Le générateur était de marque
Frésénius, Nipro, Gambro ou Bellco, selon le centre d'hémodialyse. Tous les patients en dialyse péritonéale faisaient la méthode de la dialyse péritonéale continue ambulatoire et procédaient à 4 échanges par jour.

Le diagnostic des affections cutanées était par un dermatologue et basé sur l'interrogatoire, l'examen dermatologique complet et les explorations paracliniques. Les paramètres biologiques étaient recueillis à partir des dossiers médicaux des patients.

Au cours de cette étude tous nos malades ont été interrogés et examinés par la même personne (un dermatologue) afin de standardiser les résultats. La saisie et l'analyse des données étaient réalisées avec le logiciel SPSS1. Le test utilisé pour les comparaisons était le khi-2, considéré comme statistiquement significatif lorsque p est inférieur à 0,05 .

\section{RÉSULTATS}

Durant la période d'étude, nous avons recensé 346 patients dialysés dans les différents centres de dialyse. Parmi ces patients, 208 avaient des manifestations dermatologiques soit 60,11\% (Fig. 1). Le nombre de malades en hémodialyse était de 197 (94,7\%) et de 11 (5,2\%) en dialyse péritonéale. Ils se répartissaient en 119 hommes et 89 femmes soit un sex-ratio de 1,8. Lâge moyen était de 47,23 ans avec des extrêmes de 12 à 92 ans. La tranche d'âge située entre 41 et 60 ans était la plus représentée avec $47,11 \%$. Une atopie personnelle était notée dans 41 cas $(19,7 \%)$.

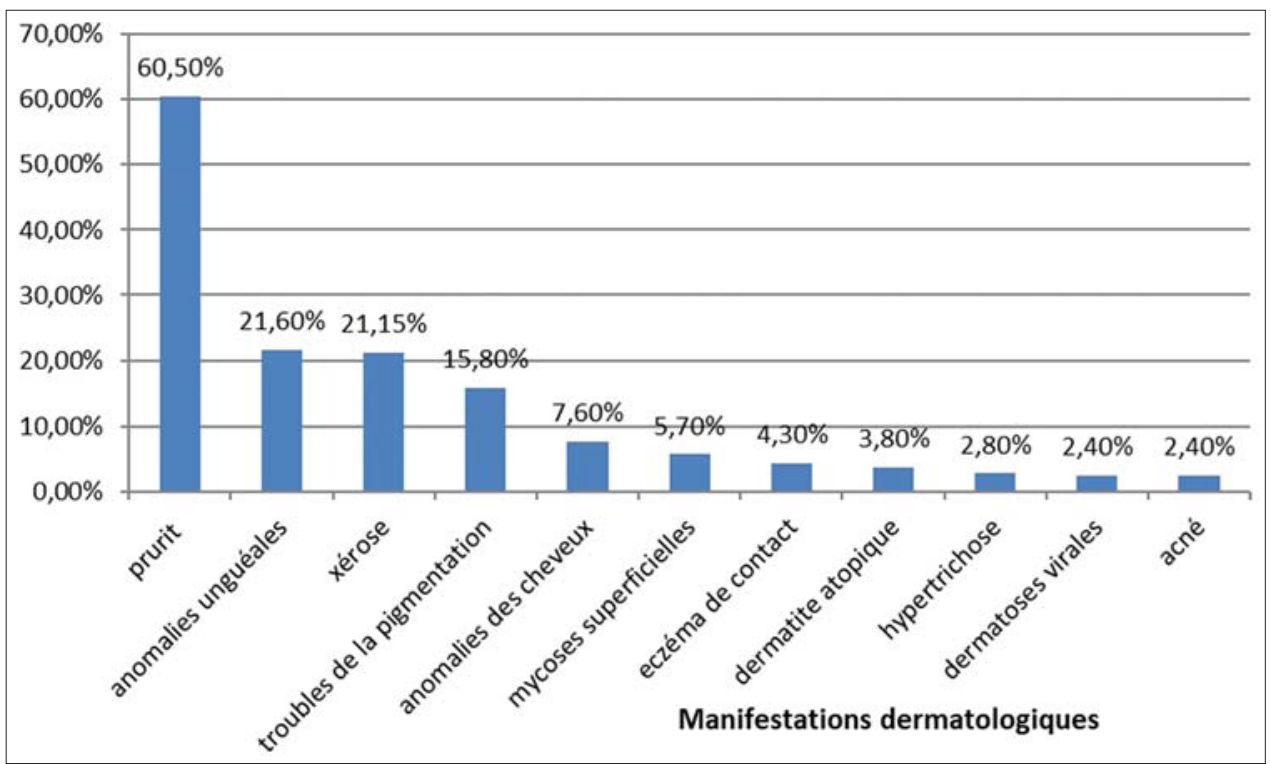

Figure 1: Représentation des Manifestations dermatologiques chez les patients dialysés. [Representation of dermatological manifestations in dialysis patients].

(C) Our Dermatol Online Supp. 1.2020 
A la clinique, le prurit observé dans 126 cas $(60,5 \%)$, était apparu durant les 6 premiers mois de dialyse dans 57 cas (45,23\%), 6 mois après l'instauration de la dialyse dans 46 cas $(36,5 \%)$ et avant la mise en route de la dialyse dans 22 cas $(17,4 \%)$. Les autres caractéristiques du prurit sont résumées dans le Tableau 1.

La xérose cutanée, observée dans 44 cas $(21,1 \%)$, était diffuse dans 21 cas $(47,72 \%)$, localisée dans 23 cas $(52,2 \%)$ avec une disposition sur les extrémités dans 14 cas $(60,8 \%)$ et sur le tronc dans 9 cas $(39,1 \%)$. Elle était associée à une ichtyose dans 12 cas $(27,27 \%)$. La durée d'évolution de la xérose était précisée dans 32 cas $(72,72 \%)$. Elle était apparue avant la dialyse dans 10 cas $(31,25 \%)$, plus de 6 mois après l'instauration de la dialyse dans 7 cas $(25 \%)$ et dans les 6 premiers mois de dialyse dans 15 cas $(43,7 \%)$.

Des anomalies unguéales étaient observées dans 45 cas $(21,6 \%)$. La durée d'évolution des onychopathies était précisée dans 16 cas. Elles étaient apparues après la mise en route de la dialyse dans 11 cas et avant la mise en route de la dialyse dans 5 cas. Les anomalies unguéales sont décrites dans le Tableau 2. On notait une anomalie des cheveux et poils d'installation progressive après l'instauration de la dialyse dans 22 cas $(10,5 \%)$. Les anomalies des cheveux et poils notées sont représentées dans le Tableau 3. Les caractéristiques des autres dermatoses de notre série, sont répertoriées dans le Tableau 4.

Tableau 1: Répartition des cas en fonction de l'horaire du prurit [Distribution of cases according to the pruritus schedule]

\begin{tabular}{lcc}
\hline Horaire du prurit & Effectif & $\%$ \\
\hline Sans horaire fixe & 51 & 40,4 \\
Prédominance nocturne & 36 & 28,5 \\
Lors de la dialyse & 20 & 15,8 \\
Après la dialyse & 12 & 9,4 \\
A la transpiration & 12 & 9,4 \\
Permanent & 4 & 3,1 \\
Après consommation de lait & 1 & 0,7 \\
Après le bain & 1 & 0,7 \\
Horaire non précisé & 23 & 18,2 \\
\hline
\end{tabular}

Tableau 2: Répartition des cas en fonction des différentes anomalies unguéales [Distribution of cases according to different nail anomalies]

\begin{tabular}{lcc}
\hline Anomalies unguéales & Effectif & \% ( $\mathbf{n = 5 7 )}$ \\
\hline Ongles striés & 22 & 38,5 \\
Ongles équisegmentés & 14 & 24,5 \\
Ongles cassants & 7 & 12,2 \\
Sillons transversaux & 4 & 7,01 \\
Onyxis & 4 & 7,01 \\
Onychogriffose & 2 & 3,5 \\
Mélanonychie & 2 & 3,5 \\
Lichen unguéal & 1 & 1,7 \\
Koïlonychie & 1 & 1,7 \\
\hline
\end{tabular}

Sur le plan étiologique, la néphroangiosclérose (NAS) était la néphropathie causale la plus fréquente $(31,3 \%)$, suivie de la néphropathie diabétique $(5,8 \%)$, de la glomérulonéphrite chronique $(4,8 \%)$, de la polykystose rénale $(4,3 \%)$ et de la néphropathie obstétricale $(0,5 \%)$. La néphropathie causale était indéterminée dans 104 cas.

Lanalyse bi variée nous avait permis d'avoir les résultats suivants:

\section{Relation Entre Le Prurit Et L'ancienneté De La Dialyse}

La répartition des cas en fonction du prurit et de l'ancienneté de la dialyse est illustrée dans le Tableau 5.

Le prurit était plus fréquent dans les 6 premiers mois de dialyse $(85 \%)$ et la corrélation était statistiquement significative $(p=0,0001)$. Les patients qui avaient fait plus de 48 mois de dialyse avaient moins tendance à avoir un prurit avec une différence statistiquement significative $(\mathrm{p}=0,002)$.

Tableau 3: Répartition des cas en fonction des anomalies des cheveux [Distribution of cases according to hair anomalies]

\begin{tabular}{lcc}
\hline Anomalies des cheveux et poils & Nombre de cas & $\%(\mathbf{n = 2 5})$ \\
\hline Effluvium télogène & 13 & 52 \\
Hypertrichose & 6 & 24 \\
Alopécie en clairière & 3 & 12 \\
Tricopathie soyeuse & 3 & 12 \\
\hline
\end{tabular}

Tableau 4: Répartition des autres dermatoses [Ditrubution of other dermatoses]

\begin{tabular}{llc}
\hline \multicolumn{2}{l}{ Les autres dermatoses } & $\begin{array}{c}\text { Nombre de } \\
\text { cas }\end{array}$ \\
\hline Dermatite atopique & Dysidrose & 2 \\
& Kératodermie palmoplantaire & 3 \\
& Eczéma des plis & 2 \\
& Prurigo nodulaire & 3 \\
& Kératose pilaire & 1 \\
Eczéma de contact & Au sparadrap & 9 \\
Trouble de la & Hyperpigmentation progressive & 11 \\
pigmentation & zones photo exposées & 18 \\
& Hyperpigmentation diffuse & 4 \\
Dermatoses & Vitiligo & 12 \\
infectieuses & Mycoses superficielles & 5 \\
& Virales (zona, molluscum & \\
\hline
\end{tabular}

Tableau 5: Répartition du prurit selon l'ancienneté de la dialyse [Distribution of pruritus occording to the age of dialysis]

\begin{tabular}{lccc}
\hline \multirow{2}{*}{ Ancienneté de la dialyse } & \multicolumn{3}{c}{ Prurit } \\
\cline { 2 - 4 } & Non & oui \\
\hline 0-6 mois & $9(15 \%)$ & $51(85 \%)$ & $\mathrm{P}=0,0001$ \\
$7-12$ mois & $13(33,3 \%)$ & $26(66,7 \%)$ & $\mathrm{P}=0,4$ \\
$13-24$ mois & $15(45,5 \%)$ & $18(54,5 \%)$ & $\mathrm{P}=0,4$ \\
$25-48$ mois & $25(54,3 \%)$ & $21(45,7 \%)$ & $\mathrm{P}=0,2$ \\
$\geq 48$ mois & $20(66,7 \%)$ & $10(33,3 \%)$ & $\mathrm{P}=0,002$ \\
\hline
\end{tabular}




\section{Relation Entre Prurit Et Type De Dialyse}

La répartition des cas en fonction de la relation entre le prurit et le type de dialyse est détaillée sur le Tableau 6.

Le prurit était plus fréquent chez les patients hémodialysés, cependant la différence n’était pas statistiquement significative avec $\mathrm{p}=0,7$.

\section{Relation Entre Le Prurit Et Le Nombre De Séances D’hémodialyse Par Semaine}

La répartition des cas en fonction du prurit et du nombre de séances de dialyse est représentée dans le Tableau 7.

Le prurit était plus fréquent chez les patients qui faisaient 2 séances d'hémodialyse par semaine et la corrélation était statistiquement significative avec $\mathrm{p}=0,0001$.

Les patients qui faisaient 3 séances d'hémodialyse par semaine avaient moins tendance à avoir un prurit avec une différence statistiquement significative $\mathrm{p}=0,0001$.

\section{Relation Entre Le Prurit Et L'atopie Personnelle}

La répartition des cas en fonction du prurit et de l'atopie personnelle est représentée dans le Tableau 8.

Le prurit était plus fréquent chez les patients qui avaient une atopie personnelle $(72,4 \%)$ et la corrélation était statistiquement significative $\mathrm{p}=0,03$.

Tableau 6: Répartition des cas de prurit selon le type de dialyse [Distribution of pruritus occording the type of dialysis]

\begin{tabular}{lccc} 
Type de dialyse & \multicolumn{3}{c}{ Prurit } \\
\cline { 2 - 4 } & Non & Oui & \\
\hline DP & $5(45,5 \%)$ & $6(54,5 \%)$ & $\mathrm{P}=0,7$ \\
HD & $77(39,1 \%)$ & $120(60,9 \%)$ & \\
\hline
\end{tabular}

Tableau 7: Répartition des cas de prurit en fonction du nombre de séances d'hémodialyse [Distribution of pruritus according to the number of hemodialysis sessions]

\begin{tabular}{lccc}
\hline \multirow{2}{*}{ Nbre de séances/semaine } & \multicolumn{3}{c}{ Prurit } \\
\cline { 2 - 4 } & Oui & non \\
\hline 3 & $54(48,6 \%)$ & $57(51,4 \%)$ & $\mathrm{P}=0,0001$ \\
2 & $52(80 \%)$ & $13(20 \%)$ & $\mathrm{P}=0,0001$ \\
1 & $1(50 \%)$ & $1(50 \%)$ & $\mathrm{P}=0,7$ \\
irrégulier & $13(68,4 \%)$ & $6(31,6 \%)$ & $\mathrm{P}=0,6$ \\
\hline
\end{tabular}

Tableau 8: Répartition des cas de prurit selon l'existence d'atopie personnelle [Distribution of pruritus cases according to the existence of personel atopy]

\begin{tabular}{lccl}
\hline Patients & Sans prurit & Avec prurit & \\
\hline Sans atopie personnelle & $66(44 \%)$ & $84(56 \%)$ & $\mathrm{P}=0,03$ \\
Avec atopie personnelle & $16(27,6 \%)$ & $42(72,4 \%)$ & \\
\hline
\end{tabular}

\section{Relation Entre Troubles Pigmentaires Et} Ancienneté De La Dialyse

La répartition des cas en fonction de l'hyperpigmentation cutanée et l'ancienneté de la dialyse est représentée dans le Tableau 9.

L'hyperpigmentation était fréquente chez les patients qui avaient fait plus de 48 mois de dialyse et la corrélation était statistiquement significative $(\mathrm{p}=0,01)$. Durant les 6 premiers mois de dialyse, l'hyperpigmentation cutanée était exceptionnelle avec une différence statistiquement significative $(\mathrm{p}=0,001)$.

A la paraclinique, l'hémogramme réalisé chez 170 malades $(81,7 \%)$ avait mis en évidence, une anémie chez 166 malades avec un taux d'hémoglobine moyen de 8,6 g/dl (extrêmes: 4,1-16,6g/dl). Les différentes caractéristiques de l'anémie et les autres explorations biologiques sont répertoriés dans le Tableau 10 .

Sur le plan thérapeutique, la prise en charge du prurit était; un anti histaminique associé à un émollient (97 cas), un anti histaminique associé à un dermocorticoïde (12 cas), un émollient seul (13 cas) et un neuroleptique

Tableau 9: Répartition des cas d' l'hyperpigmentation cutanée selon l'ancienneté de la dialyse [Distribution of cases of cutaneous hyperpigmentation according to the age of dialysis]

\begin{tabular}{lccc}
\hline \multirow{2}{*}{ Ancienneté de la dialyse } & \multicolumn{4}{c}{ Hyperpigmentation cutanée } \\
\cline { 2 - 4 } & \multicolumn{2}{c}{ Non } & oui \\
\hline $0-6$ mois & $59(98,3 \%)$ & $1(1,7 \%)$ & $\mathrm{P}=0,001$ \\
$7-12$ mois & $33(84,6 \%)$ & $6(15,4 \%)$ & $\mathrm{P}=0,7$ \\
$13-24$ mois & $30(90,9 \%)$ & $3(9,1 \%)$ & $\mathrm{P}=0,9$ \\
$25-48$ mois & $36(78,3 \%)$ & $10(21,7 \%)$ & $\mathrm{P}=0,09$ \\
$\geq 48$ mois & $21(70,0 \%)$ & $9(30,0 \%)$ & $\mathrm{P}=0,01$ \\
\hline
\end{tabular}

Tablesu 10: Rapport des différentes explorations biologiques [Report of the different biological explorations]

\begin{tabular}{|c|c|c|}
\hline \multicolumn{2}{|c|}{ Explorations biologiques } & \multirow{2}{*}{$\begin{array}{c}\begin{array}{c}\text { Nombre de } \\
\text { cas }\end{array} \\
108 \\
\end{array}$} \\
\hline $\begin{array}{l}\text { Hémogramme } \\
\text { ( } \mathrm{Hb} \text { moyen: } 8 \mathrm{~g} / \mathrm{dl})\end{array}$ & $\begin{array}{l}\text { Anémie normochrome } \\
\text { normocytaire }\end{array}$ & \\
\hline & Anémie microcytaire & 21 \\
\hline & Hypochrome normocytaire & 10 \\
\hline & Hypochrome microcytaire & 8 \\
\hline \multirow[t]{2}{*}{ Férritinémie } & élevée & 35 \\
\hline & normale & 17 \\
\hline \multirow[t]{3}{*}{ Calcémie } & élevée & 8 \\
\hline & basse & 2 \\
\hline & normale & 141 \\
\hline \multirow[t]{3}{*}{ Phosphorémie } & élevée & 47 \\
\hline & basse & 9 \\
\hline & normale & 100 \\
\hline \multirow[t]{2}{*}{ PTH } & élevé & 15 \\
\hline & normal & 43 \\
\hline \multirow[t]{2}{*}{ Aluminémie } & élevé & 34 \\
\hline & normale & 2 \\
\hline
\end{tabular}


type Gabapentine (5 cas). Le traitement de la xérose reposait sur les émollients et kératolytiques. Le traitement des anomalies des cheveux consistait à des conseils hygiéno-diététiques dans le cadre de l'effluvium télogéne et une supplémentation en vitamines B6 (3 cas).

Lévolution du prurit après le traitement par les antis histaminiques associés aux émollients était marquée par une amélioration dans 45 cas $(35,7 \%)$, une récidive à l'arrêt du traitement dans 15 cas $(11,9 \%)$. Elle était stationnaire dans 7 cas $(5,5 \%)$. La Gabapentine, utilisée dans 5 cas, avait entraîné une régression du prurit chez les 3 patients. On notait une récidive à l'arrêt du traitement chez 2 malades. Des rémissions spontanées ont été notées dans 12 cas.

L'évolution de la xérose sous émollients et ou kératolytiques était favorable dans 24 cas $(54,54 \%)$, stationnaire dans 8 cas $(18,18 \%)$. Dans 3 cas, on notait une récidive à l'arrêt des émollients.

\section{DISCUSSION}

Les manifestations dermatologiques sont fréquentes chez les dialysés comme en témoigne notre étude qui montre une fréquence de $60,11 \%$. Ces résultats rejoignent ceux de la littérature qui en situent la fréquence générale entre 50 et $100 \%$ [2,3]. Ainsi en 2009 en Turquie, sur 100 patients hémodialysés la fréquence était de 100\% [6]. En Iran, elle était de 94,1\% sur 101 patients hémodialysés en 2005 [7]. Dans l'étude de Falodun $\mathrm{O}$, réalisée au Nigéria, la fréquence était de $89,1 \%$ sur 120 patients [4]. Lâge moyen de 47,23 ans rapporté dans notre série, rejoint les données de la littérature. Ainsi en Afrique du Nord, l'âge moyen des patients était de 50,6 ans et de 44 ans respectivement dans l'étude de Masmoudi A [8] en Tunisie et Dahbi $\mathrm{N}$ au Maroc [5].

Quant à la prédominance masculine notée dans notre série, elle a été également observée dans certaines études [7,9]. Cependant dans d'autres séries [5,6], il n'y avait pas de différence entre les hommes et les femmes.

Les manifestations cliniques sont polymorphes avec une dizaine de lésions notées dans notre série. Ce polymorphisme a été déjà souligné par plusieurs études $[4,7]$. Ainsi nous avons noté une plus grande fréquence du prurit $(60,5 \%)$, suivie des anomalies unguéales $(21,6 \%)$ et de la xérose cutanée $(21,15 \%)$.
Une plus grande fréquence du prurit variant entre 70 et $90 \%$ était rapportée dans les anciennes études. Dans l'étude réalisée par Benchikhi H. en 1999 au Maroc [10], trois quart des hémodialysés chroniques avaient un prurit lié à l'hémodialyse. En revanche, elle a nettement diminué dans les nouvelles publications, variant de 20 à $65 \%$, rejoignant ainsi les résultats de notre série. La diminution nette de la fréquence du prurit ces dernières années serait liée à l'amélioration des techniques de dialyse. D'ailleurs, selon certains auteurs, une substance mal dialysable est responsable du prurit urémique par son accumulation systémique [11]. Pour certains auteurs, la survenue du prurit n’apparaît pas liée à l'ancienneté de la dialyse [12]. Pour d'autres, il existe une relation significative entre le score du prurit et la durée de l'hémodialyse [13]. Ainsi dans notre étude, nous avons retrouvé une diminution de sa fréquence en fonction de la durée de la dialyse avec un lien statistiquement significatif $(\mathrm{p}=0,002)$.

La xérose cutanée rapportée à 21,1\% dans notre série, était similaire à celle de Hajheydari Z $(22,8 \%)$ [7]. D'autres auteurs ont trouvé une fréquence plus élevée, atteignant jusqu'à $90 \%$ des cas [2,5]. Pour certains auteurs, elle serait due à une réduction de la taille et à une anomalie fonctionnelle des glandes sudoripares eccrines $[14,15]$, parfois attribuées à l'utilisation de diurétiques [9]. Mais plusieurs facteurs semblent être intrigués tels que les troubles du métabolisme de la vitamine A et la malnutrition [9].

Des anomalies unguéales étaient observées dans 21,15\% des cas, rejoignant les résultats de Masmoudi A [8]. Des études ont indiqué des valeurs allant de $52 \%$ à $71 \%[16,17]$. Par contre, des travaux plus récents ont montré une fréquence plus élevée, allant de $86 \%$ à $96 \%$ [5,9]. Les ongles striés $(38,5 \%)$ étaient les anomalies les plus fréquentes, suivies des ongles équisegmentés $(24,5 \%)$. Nos résultats sont semblables à ceux de la littérature $[2,17]$.

Une alopécie non cicatricielle est fréquemment observée chez les patients dialysés chroniques. Nous avons trouvé une alopécie dans 7,6\% dû à un effluvium télogène dans la plupart (13 cas). Dans la littérature, la fréquence des alopécies chez les hémodialysés varie de 20 à $25 \%$ [9,18]. Létiologie peut être multifactorielle, en rapport avec la sécheresse des cheveux résultant de la malnutrition, l'hyposidérémie et l'état de stress.

Une hypertrichose a été notée dans 6 cas et une acné dans 5 cas. D'autres publications ont rapporté 
des cas d'hypertrichose $[5,7]$ et d'acné $[4,5]$. Lhypertrichose de nos patients serait imputable à la prise d'anti hypertenseur vasodilatateur contenant du Minoxidil.

Les troubles pigmentaires étaient observés dans $15,8 \%$ des cas. Ces résultats sont proches de ceux de la littérature, allant de 17 à $70 \%$ [9,19]. Par contre Dahbi N avait trouvé un taux plus élevé (94\%) [5]. Ces troubles sont souvent à type de mélanodermie, prédominant au niveau des zones découvertes et se développant très progressivement tout au long de la durée d'hémodialyse. Sa pathogénie serait une mélanogenèse accrue secondaire à des taux tissulaires élevés de MSH (Melanine Stimulating Hormone). Un autre facteur pouvant aussi majorer l'hyperpigmentation de la peau est l'hémosidérose observée surtout chez les patients recevant plusieurs transfusions sanguines [8].

Enfin, l'immunodépression explique la fréquence des dermatoses infectieuses chez les dialysés avec une fréquence de 8,1\% dans notre série. Certaines publications ont rapporté une fréquence plus élevée des dermatoses infectieuses notamment en Egypte [20] et en Tunisie [8] avec respectivement $40 \%$ et $20,4 \%$.

\section{CONCLUSION}

Les manifestations dermatologiques sont fréquentes et polymorphes chez les patients dialysés. Le prurit était l'anomalie la plus fréquemment rapportée et il y existait une corrélation statistiquement significative entre le prurit et le nombre réduit de séances d'hémodialyse, le caractère récent des séances de dialyse et l'existence d'atopie personnelle. La prise en charge par des thérapeutiques simples tels que les antihistaminiques associées aux émollients a permis une évolution favorable dans la majorité des cas.

\section{Statement of Human and Animal Rights}

All procedures followed were in accordance with the ethical standards of the responsible committee on human experimentation (institutional and national) and with the Helsinki Declaration of 1975, as revised in 2008.

\section{Statement of Informed Consent}

Informed consent was obtained from all patients for being included in the study.

\section{RÉFÉRENCES}

1. Bright R. Cases and observations illustrative of renal disease. Guys Hospital Rep. 1836;1:338 -79.

2. Bencini PL, Montagnino G, Citterio A, Grazian PG, Crostia C, Ponficellih C. Cutaneous abnormalities in uremic patients. Nephron. 1985;40:316-21.

3. Guillet G. Les signes cutanés chez les hémodialysés: des marqueurs prédictifs de morbidité ou de mortalié ? Presse Med. 2006;35:377-8.

4. Falodun O, Ogunbiyi A, Salako B, George AK. Skin changes in patients with chronic renal failure. Saudi J Kidney Dis Transpl. 2011;22:268-72.

5. Dahbi N, Hocar O, Akhdari N, Amal S, Bassit N, Fadili W, et al. Manifestations cutanées chez les hémodialysés chroniques. Néphrol Thér. 2014; 10:101-5.

6. Onelmis H; Sener S, Sasmaz S, Ozer A. Cutaneous changes in patients with chronic renal failure on hemodialysis. Cutan Ocul Toxicol. 2012;31:286-91.

7. Hajheydari Z, Makhlough A. Cutaneous and Mucosal Manifestations in Patients on Maintenance Hemodialysis. A Study of 101 Patients in Sari, Iran. IJKD. 2008;2:86-90.

8. Masmoudi A, Ben Hmida M, Mseddi M, Meziou TJ, Walha N, Hachicha J, et al. Manifestations cutanées chez les hémodialysés chroniques. Étude prospective de 363 cas. Presse Med. 2006;35:399-406.

9. Deshmukh SP, Sharma YK, Dash K, Chaudhari NC, Kirti SD. Clinicoepidemiological study of skin manifestations in patients of chronic renal failure on hemodialysis. Indian Dermatol Online J. 2013;4:18-21.

10. Benchikhi H, Moussaid L, Doukaly O, Ramdani B, Zaid D, Lakhdare H. Prurit des hémodialyses chroniques: étude de 134 cas marocains. Néphrologie. 2003;23:127-31.

11. Kurban MS, Boueiz A, Kibbi AG. Cutaneous manifestations of chronic kidney disease. Clin Dermatol. 2008;26:255-64.

12. Gilchrest BA, Stern RS, Steinman A, Brown RS, Arndt KA, Anderson WW, et al. Clinical features of pruritus among patients undergoing maintenance hemodialysis. Arch Dermatol. 1982;118:154-6.

13. Szepietowski JC, Sikora M, Kusztal M, Salomon J, Magott M, Szepietowski T. Uremic pruritus: a clinical study of maintenance hemodialysis patients. J Dermatol. 2002;29:621-7.

14. Park TH, Park CH, Ha SK, Lee SH, Song KS, Lee HY, et al. Dry skin (xerosis) in patients undergoing maintenance hemodialysis: the role of decreased sweating of the eccrine sweat gland. Nephrol Dial Transplant. 1995;10:2269-73.

15. Avermaete A, Altmeyer P, Bacharach-Buhles M. Skin changes in dialysis patients: a review. Nephrol Dial Transplant. 2001;16:2293-6.

16. Altmeyer P, Kachel HG, Junger M., Koch K.M, Holzmann H, et al. Skin changes in long-term dialysis patients. Hautarzt Zeitschr Dermatol Venerol Verwand Geb. 1982;33:137-42.

17. Dyachenko P, Monelise A, Shustak A, Rozenman D. Nail Disorders in patients with chronic renal failure and undergoing haemodialysis treatment: a case control study. J Eur Acad Dermatol Venereol. 2007;23:340-4.

18. Thomas EA, Pawar B, Thomas A. A prospective study of cutaneous abnormalities in patients with chronic kidney disease. Indian J Nephrol. 2012;22:116-20.

19. Masmoudi A, Hajjaji Darouiche M, Ben Salah H, Ben Hmida M, Turki $\mathrm{H}$. Cutaneous abnormalities in patients with end stage renal failure on chronic hemodialysis. A study of 458 patients. J Dermatol Case Rep. 2014;8:86-94.

20. Sultan MM, Mansour HH, Wahby IM, Houdery AS. Cutaneous manifestations in Egyptians patients with chronic renal failure on regular hemodialysis. J Egypt Women Dermatol Soc. 2010;7:49-55.

Copyright by Maodo Ndiaye, et al. This is an open access article distributed under the terms of the Creative Commons Attribution License, which permits unrestricted use, distribution, and reproduction in any medium, provided the original author and source are credited. Source of Support: Nil, Conflict of Interest: None declared. 